ARTICLE OPEN

\title{
Tunable phonon-induced steady-state coherence in a double-quantum-dot charge qubit
}

\author{
Archak Purkayastha $\mathbb{I D}^{1 凶}$, Giacomo Guarnieri ${ }^{1}$, Mark T. Mitchison (D) $^{1}$, Radim Filip ${ }^{2 凶}$ and John Goold ${ }^{1 凶}$
}

Charge qubits can be created and manipulated in solid-state double-quantum-dot (DQD) platforms. Typically, these systems are strongly affected by quantum noise stemming from coupling to substrate phonons. This is usually assumed to lead to decoherence towards steady states that are diagonal in the energy eigenbasis. In this article, we show, to the contrary, that due to the presence of phonons the equilibrium steady state of the DQD charge qubit spontaneously exhibits coherence in the energy eigenbasis with high purity. The magnitude and phase of the coherence can be controlled by tuning the Hamiltonian parameters of the qubit. The coherence is also robust to the presence of fermionic leads. In addition, we show that this steady-state coherence can be used to drive an auxiliary cavity mode coupled to the DQD.

npj Quantum Information (2020)6:27; https://doi.org/10.1038/s41534-020-0256-6

\section{INTRODUCTION}

Preparation and coherent control of qubit states is at the heart of many quantum technologies ${ }^{1}$. Undoubtedly, quantum coherence is the most primordial non-classical effect and is at the root of many advantages displayed by quantum technologies over their classical equivalents. One of the major challenges for applications is to prepare a qubit in a state that has a controllable amount of coherence with stability in the long-time limit ${ }^{2}$. In real physical systems, quantum coherence is usually a fragile property, which is eventually destroyed by the presence of a surrounding environment $^{3,4}$. It is therefore no surprise that a plethora of strategies to preserve coherence have been conceived, such as quantum error correction $^{5}$, dynamical decoupling ${ }^{6}$ or feedback control ${ }^{7}$. All of these schemes are to some degree an inevitable battle against decoherence. Rather than fighting this battle, here we highlight a different counter-intuitive route to generate and preserve coherence using quantum noise.

We specifically consider a semiconductor double-quantum-dot (DQD) embedded on a substrate ${ }^{8-14}$ that realizes a charge qubit coupled to a phononic bath ${ }^{15,16}$. It has been previously demonstrated that properties of the DQD may be used to extract information about the phononic bath ${ }^{8,9}$. In a recent experiment ${ }^{10,11}$, the DQD and substrate were coupled to an auxiliary optical cavity, which was in turn used to experimentally characterize the spectral density of the substrate phonons. In this article, we model the dynamical evolution of these platforms and focus on their steady-state properties. Remarkably, we find that the presence of phonons autonomously drives the DQD charge qubit to a steady state that has coherence in the energy eigenbasis while retaining a significant degree of purity. This surprising result finds its explanation in the particular structure of the system-bath interaction ${ }^{17}$. Furthermore, the magnitude of steady-state coherence can be controlled by changing the experimentally tunable parameters of the qubit Hamiltonian, the detuning and the hopping. This is proven through an explicit calculation and characterization of the steady-state Bloch vector of the charge qubit as a function of the controllable Hamiltonian parameters. We also show that the coherence is robust to the presence of fermionic leads.

In addition to the obvious importance of generating coherence for quantum information processing, there is currently significant interest in harnessing coherence and exploiting it as a resource in other contexts ${ }^{18}$. In particular, coherence in the energy eigenbasis has been identified as one of the key features distinguishing quantum thermodynamics from its classical counterpart ${ }^{19-21}$. For example, coherence may enhance the performance of quantum refrigerators ${ }^{22-25}$ and heat engines ${ }^{26-28}$ or be directly converted into work $^{29-31}$. To address this point, we conclude the article by showing that, in our set-up, the above phonon-induced steadystate coherence of the DQD can in fact be exploited to drive a mode of the surrounding cavity.

\section{RESULTS}

Autonomous generation of steady-state coherence

The main theoretical idea behind the autonomous generation of steady-state coherence was first introduced and explored in ref. ${ }^{17}$. In that work, sufficient conditions concerning the structure of the interaction Hamiltonian between a qubit and a bosonic bath were identified that lead to steady-state coherence. In particular, it was shown that a spin-boson model with a Hamiltonian of the form

$\hat{\mathcal{H}}=\frac{\omega_{q}}{2} \hat{\sigma}_{z}+\sum_{k} \Omega_{k} \hat{b}_{k}^{\dagger} \hat{b}_{k}+\left(f_{1} \hat{\sigma}_{z}+f_{2} \hat{\sigma}_{x}\right) \sum_{k} \lambda_{k}\left(\hat{b}_{k}^{\dagger}+\hat{b}_{k}\right)$

autonomously leads to a non-zero steady-state value for $\left\langle\hat{\sigma}_{x}\right\rangle$. Here $\hat{\sigma}_{x, y, z}$ denote the usual Pauli spin operators, $f_{1}, f_{2} \neq 0$ two generic coupling constants and $\hat{b}_{k}$ is the bosonic annihilation operator of the $k$ th bath mode. The results presented in this paper stem from the crucial observation that a semiconductor DQD in contact with a phononic substrate is described exactly by a Hamiltonian of the form of Eq. (1).

\section{The DQD charge qubit}

The set-up we consider is depicted schematically in Fig. 1. The DQD comprises two fermionic modes with strong repulsive

\footnotetext{
${ }^{1}$ School of Physics, Trinity College Dublin, College Green, Dublin 2, Ireland. ${ }^{2}$ Department of Optics, Palacký University, 17. listopadu $1192 / 12,77146$ Olomouc, Czech Republic.

凶email: archak.p@tcd.ie; filip@optics.upol.cz; gooldj@tcd.ie
} 


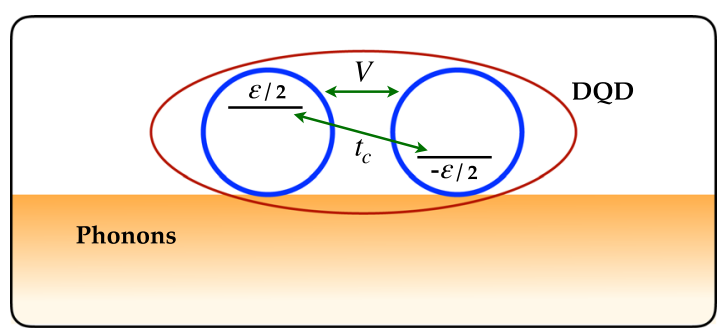

Fig. 1 Schematic depiction of two quantum dots. The two quantum dots are detuned in energy by $\varepsilon$ with inter-dot tunnelling $t_{c}$ and Coulomb repulsion $V$ interacting with a substrate supporting phononic excitations.

interaction between them. Substrate phonons are coupled to the electric dipole moment of the DQD. The full Hamiltonian (refs ${ }^{11,13,32}$ ) is then given by $\hat{\mathcal{H}}=\hat{\mathcal{H}}_{S}+\hat{\mathcal{H}}_{S E}+\hat{\mathcal{H}}_{E}$ with

$$
\begin{aligned}
\hat{\mathcal{H}}_{S}+\hat{\mathcal{H}}_{S E} & =\left(\frac{\varepsilon}{2}+\hat{B}\right)\left(\hat{n}_{1}-\hat{n}_{2}\right)+t_{c}\left(\hat{c}_{1}^{\dagger} \hat{c}_{2}+\hat{c}_{2}^{\dagger} \hat{c}_{1}\right)+V \hat{n}_{1} \hat{n}_{2}, \\
\hat{B} & =\sum_{k} \lambda_{k}\left(\hat{b}_{k}^{\dagger}+\hat{b}_{k}\right), \quad \hat{\mathcal{H}}_{E}=\sum_{k} \Omega_{k} \hat{b}_{k}^{\dagger} \hat{b}_{k},
\end{aligned}
$$

$\hat{\mathcal{H}}_{S}=\frac{\varepsilon}{2}\left(\hat{n}_{1}-\hat{n}_{2}\right)+t_{c}\left(\hat{c}_{1}^{\dagger} \hat{c}_{2}+\hat{c}_{2}^{\dagger} \hat{c}_{1}\right)+V \hat{n}_{1} \hat{n}_{2}, \hat{\mathcal{H}}_{S E}=\hat{B}\left(\hat{n}_{1}-\hat{n}_{2}\right)$.

Here $\hat{n}_{\ell}=\hat{c}_{\ell}^{\dagger} \hat{c}_{\ell}, \hat{c}_{\ell}$ is the fermionic annihilation operator of the $\ell$ th site and $\hat{b}_{k}$ is the phononic annihilation operator of the $k$ th mode of the bath. The experimentally controllable parameters of the DQD are the detuning $\varepsilon$ and the hopping $t_{c}$. The repulsive interaction between the two sites is given by $V$, which is usually much larger than any other energy scale in the regime of operation. The operator $\hat{B}$ embodies the noisy detuning due to the fluctuating phonon bath. Assuming that the latter is in a thermal state relative to an inverse temperature $\beta$, i.e. $\rho_{E}=Z_{E}^{-1} \exp \left(-\beta \hat{\mathcal{H}}_{E}\right)$ $\left(Z_{E}=\operatorname{Tr}_{E}\left(\exp \left(-\beta \hat{\mathcal{H}}_{E}\right)\right)\right)$, the noise is characterized by zero mean value, i.e. $\langle\hat{B}(t)\rangle_{E}=0$, and the auto-correlation function

$$
\langle\hat{B}(t) \hat{B}(0)\rangle_{E}=\int_{0}^{\infty} d \omega\left[W_{s}(\omega, \beta) \cos (\omega t)-i W_{a}(\omega) \sin (\omega t)\right]
$$

$W_{s}(\omega, \beta)=\mathfrak{I}_{p h}(\omega) \operatorname{coth}\left(\frac{\beta \omega}{2}\right), W_{a}(\omega)=\mathfrak{I}_{p h}(\omega)$

where $\mathfrak{I}_{p h}(\omega)=\sum_{k} \lambda_{k}^{2} \delta\left(\omega-\Omega_{k}\right)$ is the spectral function of the phononic bath and $\langle\ldots\rangle_{E}=\operatorname{Tr}\left(\rho_{E} ..\right)$. In the above equation, $W_{s}(\omega, \beta)$ and $W_{a}(\omega)$ represent the symmetric and the anti-symmetric parts of the noise power spectral density, respectively. The presence of $W_{a}(\omega)$ is the hallmark of 'quantum noise ${ }^{\prime 33}$. Phenomenologically setting $W_{a}(\omega)=0$ models 'classical noise'. This, for example, is approximately the case at high temperatures, where $W_{a}(\omega)$ is negligible compared to $W_{s}(\omega, \beta)$.

The system Hamiltonian $\hat{\mathcal{H}}_{s}$ can be diagonalized by transforming to the fermionic operators $\hat{A}_{a}$, which are related to $\hat{c}_{\ell}$ via the following transformation,

$$
\begin{aligned}
& \left(\begin{array}{l}
\hat{A}_{1} \\
\hat{A}_{2}
\end{array}\right)=\Phi\left(\begin{array}{l}
\hat{C}_{1} \\
\hat{c}_{2}
\end{array}\right), \Phi=\left(\begin{array}{cc}
\cos \left(\frac{\theta}{2}\right) & \sin \left(\frac{\theta}{2}\right) \\
-\sin \left(\frac{\theta}{2}\right) & \cos \left(\frac{\theta}{2}\right)
\end{array}\right), \\
& \theta=\tan ^{-1}\left(\frac{2 t_{c}}{\varepsilon}\right) .
\end{aligned}
$$

In the transformed basis, we have

$$
\begin{aligned}
& \hat{\mathcal{H}}_{S}=\frac{\omega_{q}}{2}\left(\hat{N}_{1}-\hat{N}_{2}\right)+V \hat{N}_{1} \hat{N}_{2}, \omega_{q}=\sqrt{\varepsilon^{2}+4 t_{c}^{2}}, \\
& \hat{\mathcal{H}}_{S E}=\left[\cos \theta\left(\hat{N}_{1}-\hat{N}_{2}\right)-\sin \theta\left(\hat{A}_{1}^{\dagger} \hat{A}_{2}+\hat{A}_{2}^{\dagger} \hat{A}_{1}\right)\right] \hat{B}, \\
& \hat{N}_{1}=\hat{A}_{1}^{\dagger} \hat{A}_{1}, \hat{N}_{2}=\hat{A}_{2}^{\dagger} \hat{A}_{2} .
\end{aligned}
$$

The DQD can be operated in the single-particle regime, i.e. where $\hat{N}_{1}+\hat{N}_{2}=1$. In this regime, the fermionic operators can be exactly mapped to Pauli spin operators,

$\hat{\sigma}_{z}=\hat{N}_{1}-\hat{N}_{2}, \hat{\sigma}_{x}=\hat{A}_{1}^{\dagger} \hat{A}_{2}+\hat{A}_{2}^{\dagger} \hat{A}_{1}$,

and $\hat{\sigma}_{y}=i\left[\hat{\sigma}_{x}, \hat{\sigma}_{z}\right] / 2=-i\left(\hat{A}_{1}^{\dagger} \hat{A}_{2}-\hat{A}_{2}^{\dagger} \hat{A}_{1}\right)$. The resulting Hamiltonians for the DQD and the DQD-phonon couplings become, in the eigenbasis of the DQD Hamiltonian,

$$
\hat{\mathcal{H}}_{S}=\frac{\omega_{\mathrm{q}}}{2} \hat{\sigma}_{z}, \hat{\mathcal{H}}_{S E}=\left(f_{1} \hat{\sigma}_{z}+f_{2} \hat{\sigma}_{x}\right) \hat{B}
$$

where $f_{1}=\cos \theta=\varepsilon / \omega_{q}$ and $f_{2}=-\sin \theta=-2 t_{c} / \omega_{q}$. The above equation describes a DQD charge qubit coupled to a phononic bath. Whatever state the charge qubit is prepared in, due to the phonons, the charge qubit relaxes to a unique steady state. To determine the latter, we notice that the above Hamiltonian shares exactly the same form as Eq. (1). This means that, in a solid-state DQD charge qubit, the presence of phonons can autonomously generate coherence in the eigenbasis of the system Hamiltonian.

In general, the quantum dots experience correlated noise due to their coupling to a common phonon bath. However, this feature is not necessary for the mechanism of coherence generation considered here, in contrast to schemes for bath-induced entanglement production where environmental correlations are essential ${ }^{34-37}$. Indeed, steady-state coherences would arise even if each quantum dot were coupled to its own independent bath, as shown in Supplementary Notes. The essential ingredient for our study is the competition between the hopping between the dots and the local coupling of each quantum dot to the bath. This condition ensures that $\hat{\mathcal{H}}_{\mathrm{SE}}$ contains both $\hat{\sigma}_{z}$ and $\hat{\sigma}_{x}$ components, as required for the autonomous generation of steady-state coherence $^{17}$. We now proceed to characterize this coherence and investigate its engineering and tunability via the DQD parameters $\varepsilon$ and $t_{c}$.

\section{Steady-state properties}

The state of a qubit is completely characterized by the expectation values of the three Pauli operators $\hat{\sigma}_{x, y, z}$. In the long-time limit, the entire set-up reaches an equilibrium steady state where the charge current, which is proportional to $\left\langle\hat{\sigma}_{y}\right\rangle$, is zero. The remaining two non-zero expectation values are found to be (see 'Methods')

$$
\begin{aligned}
& \left\langle\hat{\sigma}_{x}\right\rangle=-\frac{\sin 2 \theta}{\omega_{q}}\left[-\Delta_{s}\left(\omega_{q}, \beta\right)\left\langle\hat{\sigma}_{z}\right\rangle_{0}+\Delta_{a}\left(\omega_{q}\right)-\Delta_{a}(0)\right], \\
& \left\langle\hat{\sigma}_{z}\right\rangle=\left\langle\hat{\sigma}_{z}\right\rangle_{0}+\sin ^{2} \theta\left[\Delta_{-}\left(\omega_{q}\right)+\Delta_{+}\left(\omega_{q}\right)-\frac{\beta}{2} \operatorname{sech}^{2}\left(\frac{\beta \omega_{q}}{2}\right) \Delta_{s}\left(\omega_{q}, \beta\right)\right],
\end{aligned}
$$

where $\left\langle\hat{\sigma}_{z}\right\rangle_{0}=-W_{a}\left(\omega_{q}\right) / W_{s}\left(\omega_{q}, \beta\right)=-\tanh \left(\beta \omega_{q} / 2\right)$ and $\Delta_{s}$ $\left(\omega_{q}, \beta\right), \Delta_{a}\left(\omega_{q}\right)$ and $\Delta_{ \pm}\left(\omega_{q}, \beta\right)$ are the principal-value integrals 
defined below:

$$
\begin{aligned}
& \Delta_{s}\left(\omega_{q}, \beta\right)=\mathcal{P} \int_{0}^{\infty} \mathrm{d} \omega W_{s}(\omega, \beta)\left[\frac{1}{\omega+\omega_{q}}-\frac{1}{\omega-\omega_{q}}\right], \\
& \Delta_{a}\left(\omega_{q}\right)=\mathcal{P} \int_{0}^{\infty} \mathrm{d} \omega W_{a}(\omega)\left[\frac{1}{\omega+\omega_{q}}+\frac{1}{\omega-\omega_{q}}\right], \\
& \Delta_{ \pm}\left(\omega_{q}, \beta\right)=-\mathcal{P} \int_{0}^{\infty} \mathrm{d} \omega \frac{\left\langle\hat{\sigma}_{z}\right\rangle_{0} W_{s}(\omega, \beta) \mp W_{a}(\omega)}{\left(\omega \pm \omega_{q}\right)^{2}} .
\end{aligned}
$$

These results show that, if $\sin 2 \theta \neq 0$, which corresponds to $\varepsilon, t_{c} \neq 0$, and if the noise is quantum, i.e. $W_{a}(\omega) \neq 0$, we have $\left\langle\hat{\sigma}_{x}\right\rangle \neq 0$. Thus, in this case, there will be coherence in the eigenbasis of the system in the equilibrium steady state. If, on the other hand, the noise were classical, i.e. $W_{a}(\omega)=0$, we would obtain $\left\langle\hat{\sigma}_{x}\right\rangle=0$ and there would be no steady-state coherence. This implies that, in the high-temperature regime that corresponds to the classical limit ${ }^{33}$, no steady-state coherence will be generated. This can also be checked by taking $\beta \rightarrow 0$ limit of the above results. In the opposite limit of low temperatures, $\beta \omega_{q} \gg 1$, the above formulae simplify to

$$
\begin{aligned}
& \left\langle\hat{\sigma}_{x}\right\rangle=-2 \sin 2 \theta \int_{0}^{\infty} d \omega \frac{\mathfrak{J}_{p h}(\omega)}{\omega\left(\omega+\omega_{q}\right)} \\
& \left\langle\hat{\sigma}_{z}\right\rangle=-1+2 \sin ^{2} \theta \int_{0}^{\infty} d \omega \frac{\mathfrak{J}_{p h}(\omega)}{\left(\omega+\omega_{q}\right)^{2}} .
\end{aligned}
$$

The above equations show that, within this temperature regime, the state of the DQD becomes temperature independent. Note, however, that these expressions are only valid for temperatures well above the Kondo scale $T_{K}$, where our perturbative analysis is expected to break down ${ }^{38-40}$. Nevertheless, $T_{K}$ is exponentially suppressed by weak system-bath coupling, thus providing a wide temperature regime where our results hold.

\section{Numerical results}

Above we have found that quantum noise due to phonons can be used to generate coherence in the energy eigenbasis of the DQD charge qubit for non-zero detuning. This is shown in Fig. 2, where the dynamics of $\left\langle\hat{\sigma}_{x}(t)\right\rangle$ and $\left\langle\hat{\sigma}_{y}(t)\right\rangle$ are plotted for two different cases showing the effects of decoherence (for $\varepsilon=0$ ) and coherence generation (for $\varepsilon=1$ ) (see 'Methods'). In our calculations, we have taken the phonon spectral function as

$$
\mathfrak{J}_{p h}(\omega)=\gamma_{b} \omega\left[1-\operatorname{sinc}\left(\frac{\omega}{\omega_{c}}\right)\right] e^{-\omega^{2} / 2 \omega_{\max }^{2}} \text {. }
$$

The spectral functions of phonons in solid-state DQDs have been well characterized theoretically and experimentally and they vary depending on the experimental platform ${ }^{8-11,16}$. Our chosen spectral function is known to be a good description of bulk acoustic phonons in GaAs DQDs ${ }^{8,9,11}$. The frequency $\omega_{\max }$ is the upper cut-off frequency, while $\omega_{c}=c_{s} / d$, where $c_{s}$ is the speed of sound in the substrate and $d$ is the distance between the two quantum dots. The dimensionless parameter $\gamma_{b}$ controls the strength of coupling with phonons. The validity of our theory requires that $\gamma_{b} \ll 1$. We have set $\omega_{c}=1$ and used this as our unit of energy.

In Fig. 3, we characterize the steady state of the qubit. Since $\left\langle\hat{\sigma}_{y}\right\rangle=0$, we parameterize the steady state with $\left\langle\hat{\sigma}_{x}\right\rangle$ and the length of the Bloch vector

$r=\sqrt{\left\langle\sigma_{x}\right\rangle^{2}+\left\langle\sigma_{z}\right\rangle^{2}}$.

While $\left\langle\hat{\sigma}_{x}\right\rangle$ is a measure of coherence, $r$ is a measure of purity of the state. For a pure state, $r=1$, for a completely mixed state, $r=0$. Figure 3 demonstrates that tunable steady-state coherence persists with high purity across a range of temperatures and energy scales. The maximal value of $\left\langle\hat{\sigma}_{x}\right\rangle$ can be achieved at low temperatures by setting the dots' detuning to $\varepsilon= \pm 2 t_{c}$. The sign of the coherence can be made positive or negative, depending on the sign of $\sin 2 \theta=4 \varepsilon t_{c} / \omega_{q^{\prime}}^{2}$ as can also be seen directly from Eq. (9). Interestingly, the coherence and purity remain constant as the temperature grows up to values of order $k_{B} T \sim 0.1 \omega_{q}$, and then decay to zero as the temperature is further increased. It should therefore be possible to observe maximal phonon-induced steady-state coherence in a DQD system at only moderately low temperatures.

Let us make an order-of-magnitude estimate in order to demonstrate the feasibility of our proposal. We take $\omega_{q}=0.5 \omega_{c}$ $\varepsilon=2 t_{c}$ and $\beta \omega_{q}=10$, corresponding to the maximum coherence in Fig. 3. In GaAs, $c_{s} \approx 3000 \mathrm{~m} / \mathrm{s}$, so that an inter-dot separation of $d=150 \mathrm{~nm}$ yields $\omega_{c} \approx 20 \mathrm{GHz}$. Therefore, the maximal coherence is obtained when $\varepsilon \approx 29 \mu \mathrm{eV}, t_{c} \approx 15 \mu \mathrm{eV}$ and $T \approx 50 \mathrm{mK}$. These parameters are completely within reach of current experiments (for example, see refs ${ }^{10,11}$ ).

Robustness to the presence of fermionic leads

Experimental set-ups such as refs ${ }^{10,11}$ typically feature fermionic leads coupled to the DQD. In this case, even when the leads are in equilibrium, the number of particles in the DQD is not strictly conserved. However, the DQD can only be considered as a qubit in the single-particle sector. Nevertheless, we now show that steadystate coherence is robust to the presence of fermionic leads at equilibrium, i.e. at equal chemical potential $\mu$, and at the same temperature at the substrate phonons.

The total Hamiltonian of the set-up is now given by $\hat{\mathcal{H}}=\hat{\mathcal{H}}_{S}+$ $\hat{\mathcal{H}}_{S E}+\hat{\mathcal{H}}_{E}+\hat{\mathcal{H}}_{S f}+\hat{\mathcal{H}}_{f}, \hat{\mathcal{H}}_{S f}=\sum_{\ell=1,2} \sum_{r=1}^{\infty} \gamma_{r \ell}\left(\hat{c}_{\ell}^{\dagger} \hat{B}_{\ell r}+\hat{B}_{\ell r}^{\dagger} \hat{c}_{\ell}\right), \hat{\mathcal{H}}_{f}=$ $\sum_{\ell=1,2} \sum_{r=1}^{\infty} \mathcal{E}_{\ell r} \hat{B}_{\ell r}^{\dagger} \hat{B}_{\ell r}$, where the fermionic lead is modelled by infinite number of fermionic modes, and $\hat{B}_{\ell r}$ is the annihilation operator for the $r$ th mode of the fermionic lead attached to the $\ell$ th DQD site. For simplicity, we assume that the leads have identical, constant spectral functions $\mathfrak{J}_{\ell}^{f}(\omega)=2 \pi \sum_{r=1}^{\infty} \gamma_{\ell r}^{2} \delta\left(\omega-\mathcal{E}_{\ell r}\right)$, $\mathfrak{J}_{1}^{f}(\omega)=\mathfrak{J}_{2}^{f}(\omega)=\Gamma$, for $-\Lambda \leq \Gamma \leq \Lambda$, and zero otherwise. Here we assume that the coupling to the leads $\Gamma$ is weak, while the bandwidth $\Lambda$ is assumed to be large.

At low temperatures, the number of particles in the DQD in the equilibrium steady state is governed by the chemical potential of the fermionic lead. The DQD is occupied by a single particle on average if the chemical potential is in the regime

$-\frac{\omega_{q}}{2} \ll \mu \ll \frac{\omega_{q}}{2}+v$,

i.e. the chemical potential is higher than $\omega_{\mathrm{q}} / 2$ but smaller than the charging energy of the DQD. Therefore, when Eq. (18) holds, we expect the system to display steady-state coherence if there is non-zero detuning.

To check this, we calculate $\operatorname{Re}\left(\left\langle\hat{A}_{1}^{\dagger}(t) \hat{A}_{2}(t)\right\rangle\right)$, which corresponds to $\left\langle\hat{\sigma}_{x}(t)\right\rangle$ in the single-particle sector (see 'Methods'). The dynamics of this quantity for three different initial conditions of the DQD are shown in Fig. 4a. The three initial conditions correspond to the DQD being doubly occupied, the DQD being completely unoccupied and the completely mixed state of the charge qubit, which corresponds to $\left\langle\hat{N}_{1}(0)\right\rangle=0.5,\left\langle\hat{N}_{2}(0)\right\rangle=0.5$. None of the initial states contain any coherence in the energy eigenbasis. The chemical potential of the fermionic lead is chosen as $\mu=0$, which satisfies Eq. (18). For non-zero detuning, $\operatorname{Re}\left(\left\langle\hat{A}_{1}^{\dagger}(t) \hat{A}_{2}(t)\right\rangle\right)$ goes to the same steady-state value given by the expression for $\left\langle\hat{\sigma}_{x}\right\rangle$ in Eq. (14) for all initial conditions. Figure $4 \mathrm{~b}$ shows the plot of the equilibrium steady-state value of $\operatorname{Re}\left(\left\langle\hat{A}_{1}^{\dagger} \hat{A}_{2}\right\rangle\right)$ as a function of $\mu$. In the regime corresponding to Eq. (18), there is coherence in the energy eigenbasis, while beyond that regime, the coherence decays to zero. Thus we have shown that the coherence generated owing to the presence of phonons is robust 

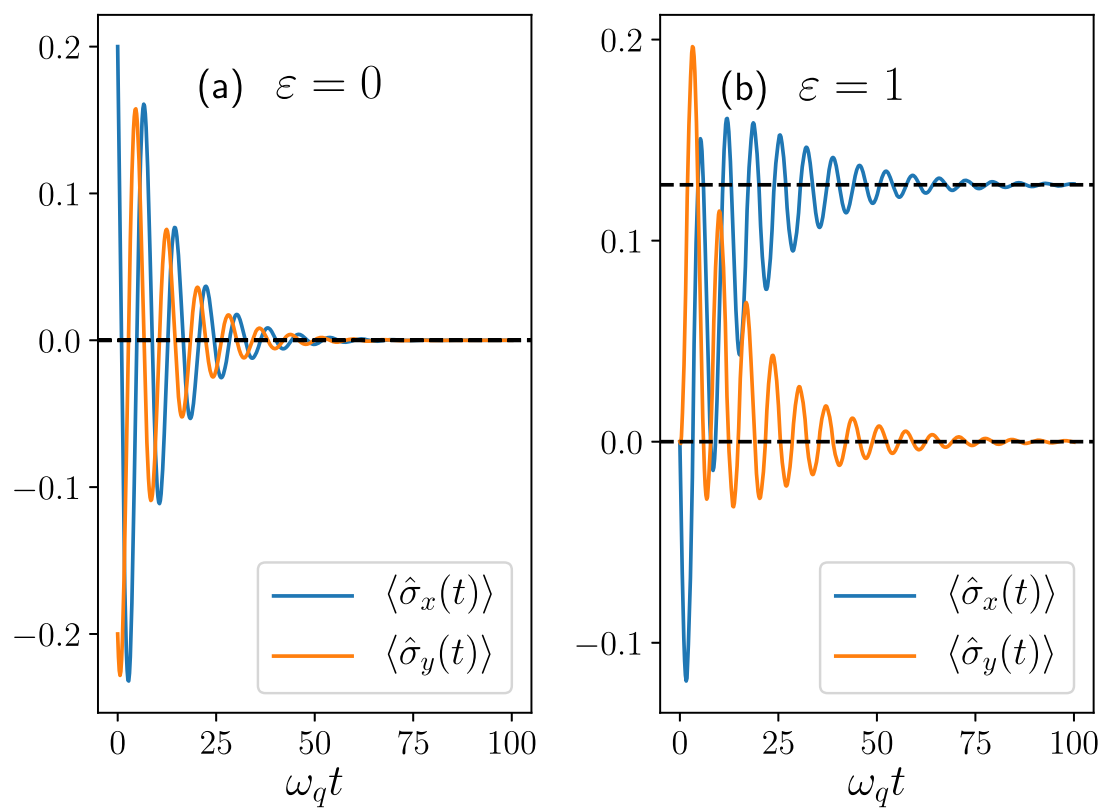

Fig. 2 Decoherence and coherence generation in DQD charge qubit due to the presence of a phononic environment. a Decoherence: dynamics of $\left\langle\hat{\sigma}_{x}(t)\right\rangle$ and $\left\langle\hat{\sigma}_{y}(t)\right\rangle$ for $\varepsilon=0$, starting from an initial state with coherence $\left\langle\hat{\sigma}_{x}(0)\right\rangle=0.2,\left\langle\hat{\sigma}_{y}(0)\right\rangle=-0.2,\left\langle\hat{\sigma}_{z}(0)\right\rangle=0$. Here the final steady state has no coherence. b Coherence generation: dynamics of $\left\langle\hat{\sigma}_{x}(t)\right\rangle$ and $\left\langle\hat{\sigma}_{y}(t)\right\rangle$ for $\varepsilon=1$, starting from a completely mixed initial state: $\left\langle\hat{\sigma}_{x}(0)\right\rangle=0,\left\langle\hat{\sigma}_{y}(0)\right\rangle=0,\left\langle\hat{\sigma}_{z}(0)\right\rangle=0$. Here the final steady state has coherence. Other parameters: $t_{c}=0.5$, $\gamma_{b}=0.03$, $\omega_{\max }=10, \beta=10$. All energies are measured in units of $\omega_{c}$, which is set to 1 .
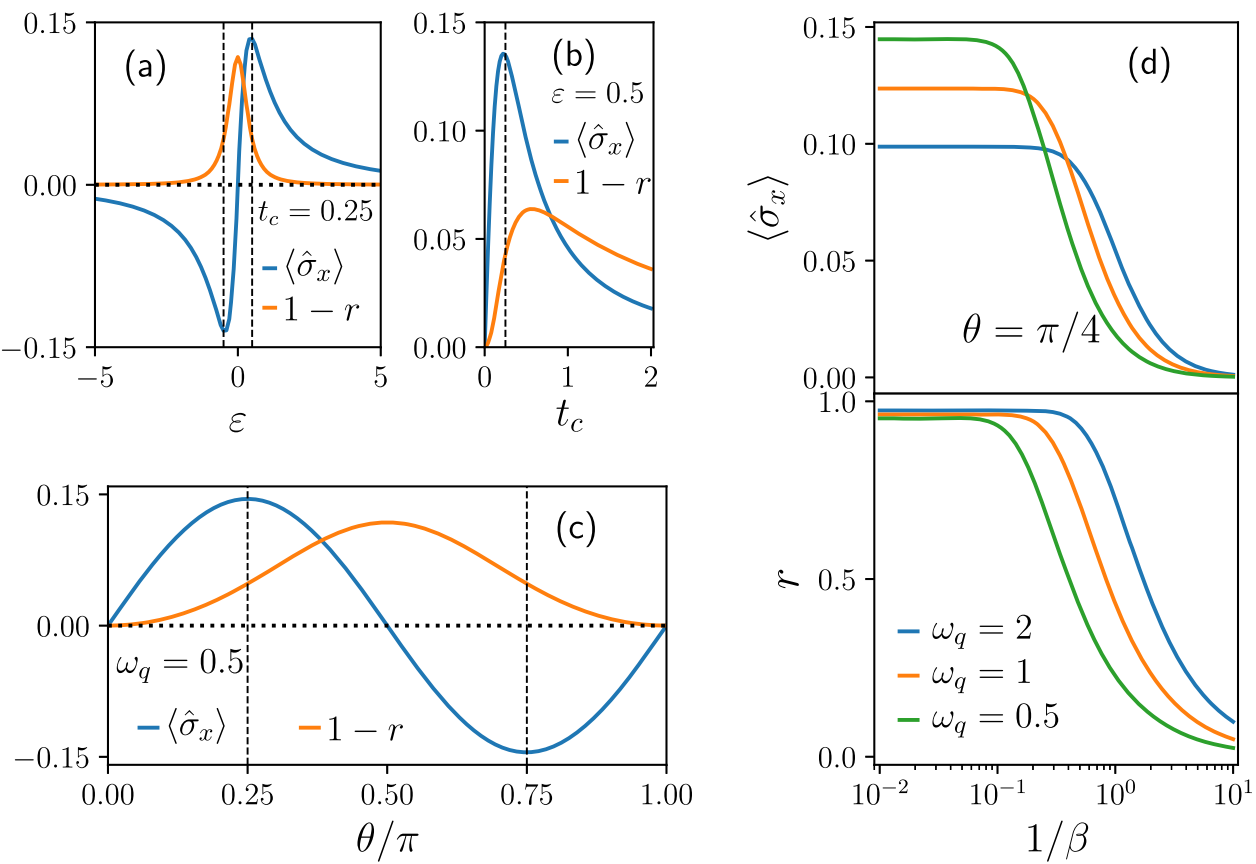

Fig. 3 The variation of $\left\langle\hat{\boldsymbol{\sigma}}_{x}\right\rangle$ and length of Bloch vector $r$ with several parameters of DQD charge qubit. a $\left\langle\hat{\sigma}_{x}\right\rangle$ and $1-r$ vs the detuning $\varepsilon$ at fixed hopping $t_{c}=0.25$ at low temperature (see Eqs 14,15$), \mathbf{b}\left\langle\hat{\sigma}_{x}\right\rangle$ and $1-r$ vs the hopping $t_{c}$ at fixed detuning $\varepsilon=0.5$ at low temperature, c $\left\langle\hat{\sigma}_{x}\right\rangle$ and $1-r$ vs $\theta$ at fixed $\omega_{q}$ at low temperature. The vertical dashed lines in these plots correspond to $\varepsilon= \pm 2 t_{c}$. d $\left\langle\hat{\sigma}_{x}\right\rangle$ (top) and $r$ (bottom) vs temperature $1 / \beta$ at fixed $\theta=\pi / 4$ and for various chosen values of $\omega_{q}$. Other parameters: $\gamma_{b}=0.03, \omega_{\max }=10$. All energies are measured in units of $\omega_{c}$, which is set to $1 .\left\langle\hat{\sigma}_{y}\right\rangle=0$.

to the presence of equilibrium fermionic leads, so long as the steady state of the DQD is in the single-particle sector.

\section{Application to driving a cavity}

As an application, we now demonstrate that phonon-induced coherence may be used to displace an auxiliary cavity mode. Let the charge qubit be coupled to a cavity mode described by a Jaynes-Cummings-like interaction

$$
\hat{\mathcal{H}}_{C}=\omega_{0} \hat{a}^{\dagger} \hat{a}, \hat{\mathcal{H}}_{S C}=g\left(\hat{\sigma}_{+} \hat{a}+\hat{a}^{\dagger} \hat{\sigma}_{-}\right) \text {. }
$$

Here $\omega_{0}$ is frequency of the cavity mode, $\hat{a}$ is bosonic annihilation operator for the cavity mode, $g$ is the cavity-DQD coupling 

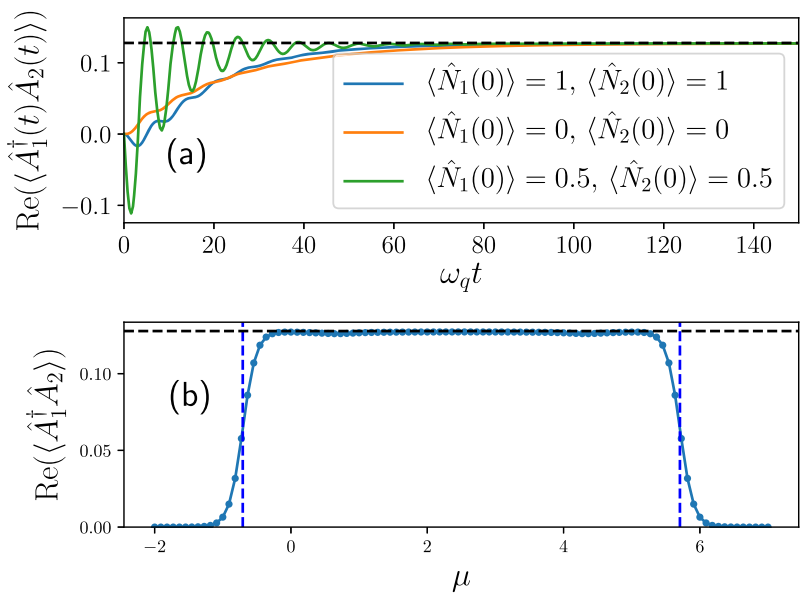

Fig. 4 The robustness of phonon-induced steady-state coherence in a DQD charge qubit to the presence of fermionic leads at equilibrium. a Evolution of $\operatorname{Re}\left(\left\langle\hat{A}_{1}^{\dagger}(t) \hat{A}_{2}(t)\right\rangle\right)$ in the presence of the fermionic lead is shown for three different initial conditions of the DQD with no initial coherence. The chemical potential of the fermionic lead is $\mu=0$. $\mathbf{b}$ The equilibrium steady-state value of $\operatorname{Re}\left(\left\langle\hat{A}_{1}^{\dagger} \hat{A}_{2}\right\rangle\right)$ as a function of $\mu$. The vertical dashed lines show positions of $\mu=-\omega_{q} / 2$ and $\mu=-\omega_{q} / 2+V$. The horizontal dashed lines in both $\mathbf{a}$ and $\mathbf{b}$ show the value of coherence given by the expression for $\left\langle\hat{\sigma}_{x}\right\rangle$ in Eq. (14). Parameters for both plots: $\varepsilon=1$, $t_{c}=0.5, \mu=0, V=5, \beta=10, \gamma_{b}=0.03, \omega_{\max }=10, \Gamma=0.06, \Lambda=400$. All energies are measured in units of $\omega_{c}$, which is set to 1 .

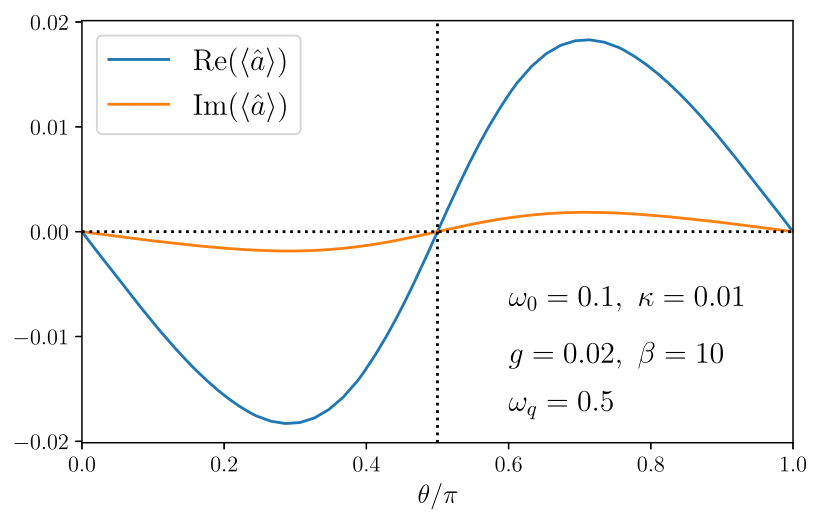

Fig. 5 A cavity with a weak Jaynes-Cummings coupling to the DQD will be displaced due to the steady-state coherence of the DQD. The figure shows plots of real and imaginary parts of $\langle\hat{a}\rangle$, as a function of DQD parameter $\theta$ with other parameters fixed. Other parameters: $\gamma_{b}=0.03, \omega_{\max }=10$. All energies are measured in units of $\omega_{c}$, which is set to 1 .

strength and $\hat{\sigma}_{ \pm}=\left(\hat{\sigma}_{x} \pm i \hat{\sigma}_{y}\right) / 2$. The cavity will be further coupled with its own thermal environment, leading to a decay rate $\kappa$.

If both the cavity-DQD coupling and the cavity decay rate are small, and if the cavity is away from resonance with the DQD (i.e. $\left.\omega_{\mathrm{q}} \neq \omega_{0}\right)$, then we can obtain the steady-state results for the cavity up to leading order in small quantities. The results for the expectation value of the cavity field operator $\langle\hat{a}\rangle$ and the cavity occupation $\left\langle\hat{a}^{\dagger} \hat{a}\right\rangle$ to leading order are given by

$$
\langle\hat{a}\rangle=-\frac{g}{2\left(\omega_{0}-i \kappa\right)}\left\langle\hat{\sigma}_{x}\right\rangle,\left\langle\hat{a}^{\dagger} \hat{a}\right\rangle \simeq \frac{1}{e^{\beta \omega_{0}}-1}+O\left(g^{2}\right),
$$

while that for $\left\langle\hat{a}^{2}\right\rangle=0$. Thus it is immediately clear that the coherence of the charge qubit causes displacement of the cavity mode, given by $\langle\hat{X}\rangle=\left\langle\hat{a}+\hat{a}^{\dagger}\right\rangle \simeq-2 g\left\langle\hat{\sigma}_{x}\right\rangle / \omega_{0}$. However, $\left\langle\hat{a}^{\dagger} \hat{a}\right\rangle$ is given by the Bose-Einstein distribution for the cavity, which ensures that no average photon current flows between the cavity and its own bosonic thermal bath. This is consistent with the fact that entire set-up is at thermal equilibrium. This means that the variance of $\hat{X}$ is given by $\operatorname{var}(\hat{X})=\left\langle\hat{X}^{2}\right\rangle-\langle\hat{X}\rangle^{2} \simeq \operatorname{coth}\left(\beta \omega_{0} / 2\right)$ $+O\left(g^{2}\right)$. The cavity displacement $\langle\hat{X}\rangle$, which may in principle be experimentally measured ${ }^{41,42}$, thus directly probes the steadystate coherence of the DQD. In Fig. 5, we show the real and imaginary parts of $\langle\hat{a}\rangle$ in steady state as the DQD parameter $\theta$ is tuned. It is clear that $\langle\hat{a}\rangle$ is non-zero when $\left\langle\hat{\sigma}_{x}\right\rangle$ is non-zero.

\section{DISCUSSION}

We have theoretically demonstrated that quantum noise due to phonons in state-of-the-art DQD charge qubits autonomously generates steady-state coherence in the energy eigenbasis of the qubit at non-zero detuning. Owing to the inherently dissipative nature of this phenomenon, it represents an especially robust yet surprisingly tunable addition to the toolbox of quantum state engineering for solid-state charge qubits and promises potential to become a new resource for quantum thermodynamics. Remarkably, the magnitude and sign of the coherence can be controlled merely by manipulating the Hamiltonian parameters of the DQD, while retaining high purity. Aside from the intrinsic interest of generating quantum coherence, the resulting steady states represent useful resources in various contexts. As a simple example, we have shown that the qubit coherence in turn generates field coherence in a cavity mode that is weakly coupled to the DQD. Moreover, coherence distillation or amplification techniques could in principle be applied to generate fully coherent resource states for quantum information processing ${ }^{43-45}$.

We emphasize that the physics described here is a general property of spin-boson models of the type in Eq. (8). Such models can generically be used to describe dissipative two-level systems $s^{4,46,47}$ and can be engineered in various platforms (for examples, see refs ${ }^{48,49}$ and citations therein). Indeed, any generic qubit Hamiltonian, $\hat{H}=\frac{\varepsilon}{2} \hat{\tau}_{z}+t_{c} \hat{\tau}_{x}$ with quantum noise in the qubit parameters $\varepsilon$ and $t_{c}$ can be written in the form of Eq. (8) (see Supplementary Notes for details). It follows that, at low temperatures, if $f_{1} f_{2} \neq 0$, which is generically true, quantum noise in qubit parameters leads to generation of steady-state coherence in the energy eigenbasis of any qubit, e.g. a superconducting qubit, a DQD spin qubit, etc. On the other hand, classical noise in qubit parameters is detrimental to such coherence. The DQD charge qubit provides an especially interesting example, since the required parameter regime corresponds to current state-of-the-art experiments ${ }^{10-14}$, where quantum noise in detuning is the major source of noise.

Future work will focus on a full characterization of thermodynamic properties of the set-up including an analysis of the relation between irreversible entropy production and coherence ${ }^{50,51}$, which will shed light on the thermodynamic cost of generating steadystate coherence.

\section{METHODS}

Detailed methods are given in Supplementary Notes. Here we give a brief overview of the techniques.

\section{Redfield master equation}

The time evolution of the DQD qubit is modelled using a Redfield master equation, detailed in Supplementary Notes. The derivation of this equation starts from a factorized initial system-environment state $\rho_{\text {tot }}(0)=\rho(0) \otimes \rho_{E \prime}$ with $\rho_{E}$ the thermal equilibrium state of the phononic bath and $\rho(0)$ the initial DQD state. The Redfield equation is based on two approximations: (i) a perturbative expansion up to second order in the coupling between the $\mathrm{DQD}$ and the reservoir, and (ii) a Markov assumption that the reservoir memory time is short in comparison to the time scale of the DQD evolution. In the presence of fermionic leads, the same set of 
approximations is used but where the leads are also incorporated into the environment. The result is a master equation describing the DQD of the form

$\frac{\partial \rho}{\partial t}=i\left[\rho, \hat{\mathcal{H}}_{S}\right]-\mathcal{L}_{p h} \rho-\mathcal{L}_{f} \rho$,

where $\mathcal{L}_{p h}$ and $\mathcal{L}_{f}$, respectively, describe the effect of the phononic and fermionic reservoirs (see Supplementary Notes). The solution of Eq. (21) is used to compute the time evolution of expectation values in the main text.

Perturbation expansion of the generalized equilibrium state As discussed in ref. ${ }^{52}$, the predictions of the Redfield equation give results for the DQD coherence valid up to second order in $\hat{\mathcal{H}}_{S E}$, while the predictions for the populations are valid only to zeroth order. However, in order to discuss the purity of the qubit, we need results for both coherences and populations valid to the same order. In order to achieve this, we use the fact that an open quantum system coupled weakly to a thermal environment is generically expected to relax to the generalized equilibrium state ${ }^{53-58}$

$\lim _{t \rightarrow \infty} \rho(t)=\operatorname{Tr}\left[\frac{\mathrm{e}^{-\beta\left(\hat{\mathcal{H}}_{S}+\hat{\mathcal{H}}_{E}+\hat{\mathcal{H}}_{S E}\right)}}{\operatorname{Tr}\left[\mathrm{e}^{-\beta\left(\hat{\mathcal{H}}_{S}+\hat{\mathcal{H}}_{E}+\hat{\mathcal{H}}_{S E}\right)}\right]}\right]$,

which incorporates the effect of system-environment correlations. We use a perturbative expansion of Eq. (22) up to second order in $\hat{\mathcal{H}}_{S E}$ to obtain steady-state expectation values, as detailed in Supplementary Notes. This approach yields an identical prediction for the coherence $\left\langle\hat{\sigma}_{x}\right\rangle$ as the master equation, as well as a second-order correction to the population inversion $\left\langle\hat{\sigma}_{z}\right\rangle$.

\section{DATA AVAILABILITY}

Our main results are analytical. The data sets generated during numerical evaluation of analytical formulas are available on reasonable request.

Received: 10 July 2019; Accepted: 7 February 2020;

Published online: 13 March 2020

\section{REFERENCES}

1. Nielsen, M. A. \& Chuang, I. Quantum Computation and Quantum Information (Cambridge University Press, 2002).

2. Bennett, C. H. \& DiVincenzo, D. P. Quantum information and computation. Nature 404, 247 (2000).

3. Alicki, R. \& Lendi, K. Quantum Dynamical Semigroups and Applications, Volume 717 of Lecture Notes in Physics (Springer, Berlin, Heidelberg, 2007).

4. Breuer, H.-P. \& Petruccione, F. The Theory of Open Quantum Systems (Oxford University Press, 2002).

5. Shor, P. W. Scheme for reducing decoherence in quantum computer memory. Phys. Rev. A 52, 2493-2496 (1995).

6. Viola, L., Knill, E. \& Lloyd, S. Dynamical decoupling of open quantum systems. Phys. Rev. Lett. 82, 2417-2421 (1999).

7. Rabitz, H., Vivie-riedle, R. D., Motzkus, M. \& Kompa, K. Whither the future of controlling quantum phenomena? Science 288, 1-5 (2005).

8. Weber, C. et al. Probing confined phonon modes by transport through a nanowire double quantum dot. Phys. Rev. Lett. 104, 036801 (2010).

9. Colless, J. I. et al. Raman phonon emission in a driven double quantum dot. Nat. Commun. 5, 3716(2014).

10. Hartke, T. R., Liu, Y.-Y., Gullans, M. J. \& Petta, J. R. Microwave detection of electronphonon interactions in a cavity-coupled double quantum dot. Phys. Rev. Lett. 120, 097701 (2018).

11. Gullans, M. J., Taylor, J. M. \& Petta, J. R. Probing electron-phonon interactions in the charge-photon dynamics of cavity-coupled double quantum dots. Phys. Rev. B 97, 035305 (2018).

12. Liu, Y.-Y. et al. On-chip quantum-dot light source for quantum-device readout. Phys. Rev. Appl. 9, 014030 (2018).

13. Stehlik, J. et al. Double quantum dot floquet gain medium. Phys. Rev. X 6, 041027 (2016).

14. Liu, Y.-Y. et al. Semiconductor double quantum dot micromaser. Science 347, 285-287 (2015).

15. Loss, D. \& DiVincenzo, D. P. Quantum computation with quantum dots. Phys. Rev. A 57, 120-126 (1998).
16. Brandes, T. Coherent and collective quantum optical effects in mesoscopic systems. Phys. Rep. 408, 315-474 (2005).

17. Guarnieri, G., Koláŕ, M. \& Filip, R. Steady-state coherences by composite systembath interactions. Phys. Rev. Lett. 121, 070401 (2018).

18. Streltsov, A., Adesso, G. \& Plenio, M. B. Colloquium: Quantum coherence as a resource. Rev. Mod. Phys. 89, 041003 (2017).

19. Lostaglio, M., Korzekwa, K., Jennings, D. \& Rudolph, T. Quantum coherence, timetranslation symmetry, and thermodynamics. Phys. Rev. X 5, 021001 (2015).

20. Lostaglio, M., Jennings, D. \& Rudolph, T. Description of quantum coherence in thermodynamic processes requires constraints beyond free energy. Nat. Commun. 6, 6383 (2015).

21. Narasimhachar, V. \& Gour, G. Low-temperature thermodynamics with quantum coherence. Nat. Commun. 6, 7689 (2015).

22. Correa, L. A., Palao, J. P., Alonso, D. \& Adesso, G. Quantum-enhanced absorption refrigerators. Sci. Rep. 4, 3949 (2014).

23. Mitchison, M. T., Woods, M. P., Prior, J. \& Huber, M. Coherence-assisted single-shot cooling by quantum absorption refrigerators. New J. Phys. 17, 115013 (2015).

24. Brask, J. B. \& Brunner, N. Small quantum absorption refrigerator in the transient regime: time scales, enhanced cooling, and entanglement. Phys. Rev. E 92, 062101 (2015).

25. Maslennikov, G. et al. Quantum absorption refrigerator with trapped ions. Nat. Commun. 10, 202 (2019).

26. Scully, M. O., Chapin, K. R., Dorfman, K. E., Kim, M. B. \& Svidzinsky, A. Quantum heat engine power can be increased by noise-induced coherence. Proc. Natl Acad. Sci. USA 108, 15097-15100 (2011).

27. Ptaszyński, K. Coherence-enhanced constancy of a quantum thermoelectric generator. Phys. Rev. B 98, 085425 (2018).

28. Klatzow, J. et al. Experimental demonstration of quantum effects in the operation of microscopic heat engines. Phys. Rev. Lett. 122, 110601 (2019).

29. Allahverdyan, A. E., Balian, R. \& Nieuwenhuizen, T. M. Maximal work extraction from finite quantum systems. EPL (Europhys. Lett.) 67, 565 (2004).

30. Korzekwa, K., Lostaglio, M., Oppenheim, J. \& Jennings, D. The extraction of work from quantum coherence. New J. Phys. 18, 023045 (2016).

31. Kammerlander, P. \& Anders, J. Coherence and measurement in quantum thermodynamics. Sci. Rep. 6, 22174 (2016).

32. Agarwalla, B. K., Kulkarni, M., Mukamel, S. \& Segal, D. Giant photon gain in largescale quantum dot-circuit qed systems. Phys. Rev. B 94, 121305 (2016).

33. Clerk, A. A., Devoret, M. H., Girvin, S. M., Marquardt, F. \& Schoelkopf, R. J. Introduction to quantum noise, measurement, and amplification. Rev. Mod. Phys. 82, 1155-1208 (2010).

34. Benatti, F., Floreanini, R. \& Piani, M. Environment induced entanglement in markovian dissipative dynamics. Phys. Rev. Lett. 91, 070402 (2003).

35. Solenov, D., Tolkunov, D. \& Privman, V. Exchange interaction, entanglement, and quantum noise due to a thermal bosonic field. Phys. Rev. B 75, 035134 (2007).

36. McCutcheon, D. P. S., Nazir, A., Bose, S. \& Fisher, A. J. Long-lived spin entanglement induced by a spatially correlated thermal bath. Phys. Rev. A 80, 022337 (2009).

37. Zell, T., Queisser, F. \& Klesse, R. Distance dependence of entanglement generation via a bosonic heat bath. Phys. Rev. Lett. 102, 160501 (2009).

38. Guinea, F. Dynamics of simple dissipative systems. Phys. Rev. B 32, 4486-4491 (1985).

39. Costi, T. A. Scaling and universality in the anisotropic kondo model and the dissipative two-state system. Phys. Rev. Lett. 80, 1038-1041 (1998).

40. Saito, K. \& Kato, T. Kondo signature in heat transfer via a local two-state system. Phys. Rev. Lett. 111, 214301 (2013).

41. Breitenbach, G., Schiller, S. \& Mlynek, J. Measurement of the quantum states of squeezed light. Nature 387, 471-475 (1997).

42. Bakker, M. P. et al. Homodyne detection of coherence and phase shift of a quantum dot in a cavity. Opt. Lett. 40, 3173-3176 (2015).

43. Winter, A. \& Yang, D. Operational resource theory of coherence. Phys. Rev. Lett. 116, 120404 (2016).

44. Liu, C. L. \& Zhou, D. L. Deterministic coherence distillation. Phys. Rev. Lett. 123, 070402 (2019).

45. Manzano, G., Silva, R. \& Parrondo, J. M. R. Autonomous thermal machine for amplification and control of energetic coherence. Phys. Rev. E 99, 042135 (2019).

46. Leggett, A. J. et al. Dynamics of the dissipative two-state system. Rev. Mod. Phys. 59, 1-85 (1987).

47. Thorwart, M., Paladino, E. \& Grifoni, M. Dynamics of the spin-boson model with a structured environment. Chem. Phys. 296, 333-344 (2004).

48. Hur, K. L. et al. Driven dissipative dynamics and topology of quantum impurity systems. C. R. Phys.19, 451-483 (2018).

49. Paladino, E., Galperin, Y. M., Falci, G. \& Altshuler, B. L. 1/f noise: implications for solid-state quantum information. Rev. Mod. Phys. 86, 361-418 (2014).

50. Santos, J. P., Céleri, L. C., Landi, G. T. \& Paternostro, M. The role of quantum coherence in non-equilibrium entropy production. npj Quantum Inf. 5, 23 (2019). 
51. Francica, G., Goold, J. \& Plastina, F. Role of coherence in the nonequilibrium thermodynamics of quantum systems. Phys. Rev. E 99, 042105 (2019).

52. Fleming, C. H. \& Cummings, N. I. Accuracy of perturbative master equations. Phys. Rev. E 83, 031117 (2011).

53. Dhar, A., Saito, K. \& Hänggi, P. Nonequilibrium density-matrix description of steady-state quantum transport. Phys. Rev. E 85, 011126 (2012).

54. Thingna, J., Wang, J.-S. \& Hänggi, P. Generalized gibbs state with modified redfield solution: exact agreement up to second order. J. Chem. Phys. 136, 194110 (2012).

55. Thingna, J., Wang, J.-S. \& Hänggi, P. Reduced density matrix for nonequilibrium steady states: a modified redfield solution approach. Phys. Rev. E 88, 052127 (2013).

56. Xu, X., Thingna, J. \& Wang, J.-S. Finite coupling effects in double quantum dots near equilibrium. Phys. Rev. B 95, 035428 (2017).

57. Subaşı, Y., Fleming, C. H., Taylor, J. M. \& Hu, B. L. Equilibrium states of open quantum systems in the strong coupling regime. Phys. Rev. E 86, 061132 (2012).

58. Alipour, S. et al. Correlation picture approach to open-quantum-system dynamics. Preprint at https://arxiv.org/abs/1903.03861 (2019).

\section{ACKNOWLEDGEMENTS}

We acknowledge funding from European Research Council Starting Grant ODYSSEY (Grant Agreement No. 758403). J.G. also acknowledges funding from a SFI Royal Society University Research Fellowship. R.F. acknowledges grant 1917765 S of Czech Science Foundation and national funding from the MEYS as well as funding from the European Union's Horizon 2020 (2014-2020) research and innovation framework programme under grant agreement No. 731473 (project 8C18003 TheBlinQC). Project TheBlinCQ has received funding from the QuantERA ERA-NET CoFund in Quantum Technologies implemented within the European Union's Horizon 2020 programme.

\section{AUTHOR CONTRIBUTIONS}

A.P. conceptualized the idea. A.P., M.T.M. and G.G. did all the analytical calculations. A.P. carried out the numerical evaluation of analytical results. R.F. and J.G. supervised the project and checked the correctness of the physical results and their impacts. All authors contributed towards writing the manuscript.

\section{COMPETING INTERESTS}

The authors declare no competing interests.

\section{ADDITIONAL INFORMATION}

Supplementary information is available for this paper at https://doi.org/10.1038/ s41534-020-0256-6.

Correspondence and requests for materials should be addressed to A.P., R.F. or J.G.

Reprints and permission information is available at http://www.nature.com/ reprints

Publisher's note Springer Nature remains neutral with regard to jurisdictional claims in published maps and institutional affiliations.

Open Access This article is licensed under a Creative Commons Attribution 4.0 International License, which permits use, sharing, adaptation, distribution and reproduction in any medium or format, as long as you give appropriate credit to the original author(s) and the source, provide a link to the Creative Commons license, and indicate if changes were made. The images or other third party material in this article are included in the article's Creative Commons license, unless indicated otherwise in a credit line to the material. If material is not included in the article's Creative Commons license and your intended use is not permitted by statutory regulation or exceeds the permitted use, you will need to obtain permission directly from the copyright holder. To view a copy of this license, visit http://creativecommons. org/licenses/by/4.0/.

(c) The Author(s) 2020 